\title{
GOBIERNO LOCAL Y CONVIVENCIA PACÍFICA: REFLEXIONES DESDE CENTROAMÉRICA Y REPÚBLICA DOMINICANA ${ }^{1}$
}

\author{
Rodolfo Calderón Umaña \\ rcalderon@flacso.or.cr \\ FLACSO, Costa Rica
}

\section{RESUMEN}

A partir de los resultados de una investigación sobre el vínculo entre gobierno local y seguridad ciudadana en Centroamérica y República Dominicana, el documento evidencia las tareas que las municipalidades pueden y deben ejecutar para contribuir a mejorar la seguridad ciudadana. El análisis revela, sin embargo, que al actuar estratégicamente sobre áreas y funciones que les son privativas, los gobiernos locales resultan ser aptos para anticipar el surgimiento de ciertas formas de violencia no criminal. De ahí que su papel trascienda la esfera de la seguridad y se instale en el ámbito de la prevención y el fomento de la convivencia pacífica.

Palabras claves: seguridad ciudadana, gobierno local, convivencia pacífica, política pública, Centroamérica. 


\section{ABSTRACT}

Based on the results of a research project that analyzed the role of local governments in the improvement of citizen security in Central America and the Dominican Republic, the article underlines the fact that municipalities could prevent effectively the emergency of certain forms of violence, when they strategically promote and strengthen functions that are constitutive of their daily routine. Hence, local governments should work preventing violence and promoting a culture of peace, not just undertake specific actions in the realm of citizen security.

Key words: citizen security, local government, culture of peace, public policy, Central America. 


\section{INTRODUCCIÓN}

Uno de los problemas que más afecta la vida de las y los latinoamericanos en la actualidad es la presencia de ciertas formas de violencia criminal. Ya en el año 2000 se estimaba que, en promedio, 140 mil personas eran asesinadas al año, 28 millones eran víctimas de robo o hurto y se perdían al menos tres días de vida saludable por cada habitante, todo ello con un costo cercano al 14\% del producto interno bruto regional (Londoño y otros 2000). Hoy, casi una década después, este problema se agudiza y encuentra en América Central una de sus expresiones más acabadas. Así lo ejemplifica el hecho de que la tasa de homicidios en la región supera en siete puntos la tasa de América Latina (PNUD 2009).

Es menester advertir, sin embargo, que no se está ante un fenómeno indiferenciado. Por el contrario, la violencia, en sus distintas manifestaciones, se ve afectada por variables como el género, la edad, el grupo socioeconómico y el área geográfica. En este sentido, la evidencia disponible muestra que los jóvenes - hombres- son las principales víctimas de delitos patrimoniales y físicos en el espacio público. Por otra parte, las mujeres son las víctimas más frecuentes de violencia en el ámbito familiar y en las relaciones de pareja, y son también las que reportan el mayor número de agresiones sexuales. Los delitos al patrimonio son más frecuentes entre los grupos de mayores ingresos y las áreas urbanas las que presentan los índices más altos de victimización (PNUD 2008).

Desde esta perspectiva, los países del área se pueden agrupar en dos grandes bloques: el llamado triángulo norte (Guatemala, El Salvador y Honduras) y el triángulo sur (Nicaragua, Costa Rica y Panamá). En el primer grupo predominan los delitos contra la vida, fundamentalmente los homicidios dolosos, cuya tasa se alinea entre las más altas del planeta. En el segundo grupo son más frecuentes los delitos contra la propiedad, sobre todo los robos con violencia contra las personas, los robos con violencia sobre las 
cosas y los hurtos. ${ }^{2}$ Sin embargo, al interior de estos países también hay diferencias notorias.

Por ejemplo, en El Salvador veinte municipios de un total de 262 concentran la violencia homicida (todos ellos responden a centros urbanos altamente poblados). En Guatemala, los municipios con predominio de población no indígena registran las mayores tasas homicidas. Y en Costa Rica, la zona del Caribe, una de las menos pobladas, reporta las mayores tasas de homicidios.

Los factores asociados al aumento de la violencia criminal en Centroamérica varían según el grupo de países. Para el triángulo norte, varios autores coinciden en que los procesos de migración masiva y forzada, originados por el conflicto bélico en la década de los ochenta y posteriormente por las dinámicas excluyentes del modelo económico de posguerra (década de los noventa), desarticularon estructuras sociales básicas como la familia y la comunidad. Con este panorama, se creó un vacío que luego fue ocupado por los grupos juveniles denominados maras, los cuales se constituyen desde sus orígenes en una red de apoyo que, ante la incapacidad del modelo social para generar oportunidades educativas y laborales, recurren al delito para su mantenimiento (Zinecker 2010 y ERIC et al. 2001). Sin embargo, es claro que ciertas manifestaciones de violencia provocadas por estos grupos como, por ejemplo, los ritos de iniciación o la venganza por deserción, poco se relacionan con la criminalidad común remitiendo, por el contrario, a procesos derivados del vínculo primario entre el joven y el grupo de referencia.

Aunque no se ha ensayado una interpretación común para los países que integran el triángulo sur, lo argumentado hasta el momento lleva a pensar que una de las principales razones del incremento delictivo -caracterizado por un predominio de infracciones de naturaleza económica — se relaciona con la tensión estructural emergida de la nueva oleada globalizadora que crea un orden social de carácter individualista. Este, por un lado, promueve determinados estándares de consumo como metas 
realizables para todos, sin importar la posición en la estructura social y, por el otro, excluye sistemáticamente a un considerable sector social de los mecanismos institucionales dirigidos a alcanzar los parámetros de bienestar material y social impuestos por la cultura. Esta tensión, unida a la exacerbación de valores como la competencia, el individualismo y la inmediatez, favorece las condiciones oportunas para el surgimiento del delito, sobre todo de orden patrimonial, como una vía, no la más común ni la más frecuente, para resolver la tensión estructuralmente inducida entre medios y expectativas (Calderón 2010).

Lamentablemente, y a pesar de la complejidad de la problemática descrita, varios elementos se han conjugado para dar paso a un abordaje unidimensional y empobrecido que acentúa la dimensión criminal del problema y magnifica el aspecto represivo frente a cualquier otra opción. Y esto, al igual que en otras regiones del mundo, ha llevado a que la respuesta institucional se aglutine alrededor de cierto tipo de delitos y siga la línea del rigor punitivo, lo que estimula el encierro como única respuesta posible y deseable. El caso extremo de esta situación se ha manifestado en el triángulo norte con sus políticas de mano dura y súper mano dura. Pero también en países como Costa Rica, que se precia de contar con una larga tradición democrática, estas acciones han puesto en entredicho libertades y garantías individuales propias de un Estado de derecho.

El problema, sin embargo, es que a pesar de lo que sostienen los defensores del credo punitivo, la tasa general de delitos en Centroamérica sigue creciendo junto con la población penitenciaria, lo que deja en claro que estas medidas no han alcanzado el resultado que se esperaba. ¿Será acaso que se necesita más represión o que esta no se ha aplicado de manera efectiva? La realidad parece sugerir lo contrario, pues no hay evidencia empírica disponible para ningún país conocido que muestre, en forma satisfactoria, que el aumento en los índices de encarcelamiento se acompaña de una disminución de la criminalidad. 
Quizás la razón de mayor peso para comprender la ineficacia de estas políticas sea el supuesto sobre el que se erige: el delito es percibido, en esencia, como un fenómeno estrictamente individual, ajeno y libre de condicionantes sociales, económicos y culturales. De ahí que el castigo y no la prevención, sobre todo el encarcelamiento, se levanta triunfante como la única respuesta ante el aumento de la criminalidad. Esta situación se constata fácilmente al observar el crecimiento de la población penitenciaria en todo el mundo, incluso en países como Noruega, caracterizados históricamente por su renuencia a dicha forma de escarmiento. En ese país, por ejemplo, el número de presos, que en 1960 no alcanzaba los cuatro por cada diez mil habitantes, en la actualidad es superior a los seis (Kliksberg 2007).

Es claro, frente a la realidad enunciada, que un abordaje estrictamente punitivo del delito, que deposita en el sistema de justicia penal toda la expectativa y la responsabilidad de generar respuestas efectivas y sostenibles ante el problema en cuestión, está condenado al fracaso. Se necesita dar un paso más allá: reconocer el carácter social del problema y, por tanto, imaginar respuestas que integren lo represivo y lo preventivo de forma adecuada, es decir, acentuar la prevención y ubicar la represión en el lugar que le corresponde en una sociedad democrática: como recurso de última instancia. Por tanto, su papel es elemental pero limitado, de ahí la necesidad de reconocer y potenciar el papel que pueden y deben asumir otros actores no solo alrededor de la prevención del delito sino de la violencia en general.

En este sentido destacan, como contrapunto a lo indicado, dos hechos primordiales que se registran en años recientes. En primer lugar, el surgimiento de un enfoque para el diseño de políticas públicas que, a diferencia del discurso y las prácticas hegemónicas, ve en el delito (como en otras formas de violencia social) un problema relacionado con el desarrollo y los derechos humanos. Por ello considera que, para abordar efectiva y democráticamente el tema, se requiere elaborar y ejecutar políticas públicas que, sin abandonar lo represivo, acentúen lo preventivo al tiempo que estimulen la participación ciudadana. Esta propuesta ha sido desarrollada por el Programa de las Naciones Unidas 
para el Desarrollo bajo el concepto de «seguridad ciudadana» que, se entiende, forma parte de la seguridad humana (PNUD 2005). ${ }^{3}$

En segundo lugar, la emergencia de nuevos actores en el tratamiento del problema, específicamente aquellos cuyo campo de acción se alinea, por excelencia, en el ámbito preventivo no sólo del delito, sino también de otras formas de violencia social. ${ }^{4}$ En este sentido, adquieren particular relevancia los gobiernos municipales puesto que algunos de ellos, en el ejercicio de sus funciones y competencias, han comenzado a generar iniciativas en este campo que pueden contribuir de manera significativa al tema si se orientan adecuadamente. ${ }^{5}$

Por ello, y conscientes de esta realidad, después de un largo proceso de investigación y reflexión sobre el tema de los gobiernos locales y su vínculo con la seguridad ciudadana en Centroamérica, se ha elaborado el presente documento. No se trata de una síntesis de los resultados del proyecto sino de una propuesta que, tomando en consideración las lecciones aprendidas del estudio y su lectura en perspectiva comparada, identifica y precisa áreas estratégicas y tareas concretas que las municipalidades pueden realizar para mejorar la seguridad ciudadana, pero sobre todo para prevenir la violencia y promover la convivencia pacífica desde lo local. ${ }^{6}$

Antes de desplegar tales propuestas se ha considerado necesario incluir una breve reseña de los aspectos teórico-metodológicos que guiaron el estudio sobre el que se estructura la reflexión, ello con el fin de enmarcar sus alcances y limitaciones. Luego se presenta una nota de advertencia sobre lo que no deberían hacer las municipalidades en este contexto y, por último, el documento analiza los desafíos y perspectivas que enfrentan las municipalidades para actuar en la línea indicada. 


\section{PRECISIONES SIGNIFICATIVAS}

\section{MARCO CONCEPTUAL}

El proyecto que da sustento a este trabajo asumió como marco de referencia el esquema analítico sobre seguridad ciudadana elaborado por el PNUD (2005), ya que éste, a pesar de sus limitaciones, constituye una valiosa herramienta para el diseño de políticas públicas. Esto, en primer lugar porque delimita con claridad su objeto de estudio: las vulnerabilidades que atentan contra un núcleo esencial de derechos fundamentales -la vida; la integridad física, emocional y sexual, y otros derechos propios del fuero personal como la inviolabilidad del domicilio, la libertad de movimiento y el disfrute del patrimonio-, lo cual prioriza acciones y focaliza recursos. En segundo lugar, el esquema analítico del PNUD, al concebir la seguridad ciudadana como un componente de la seguridad humana —aquel que atiende las vulnerabilidades causadas por el despojo y la violencia - la relaciona inevitablemente con el desarrollo social y los derechos humanos. ${ }^{7}$ Esto permite sustraer el tema del ámbito autoritario y represivo en el que lo encasillan los discursos dominantes — políticas de ley y orden- y abre el espacio para la intervención de nuevos actores desde la prevención y la participación ciudadana.

\section{ASPECTOS METODOLÓGICOS}

En virtud de que era materialmente imposible analizar todas las experiencias sobre el tema, el proyecto incluyó dos casos por país. La selección se basó en juicios de expertos (académicos, funcionarios públicos y autoridades municipales), y en lo fundamental recopila el tipo de acciones que han empezado a desarrollar los gobiernos locales en Centroamérica sobre seguridad ciudadana, prevención de la violencia y promoción de la convivencia pacífica. Las iniciativas también expresan las características distintivas de las municipalidades en la región: debilidad institucional, escasez de personal calificado, carencia de recursos materiales, y diversidad en términos de problemáticas, población y extensión territorial. 
El grado de estructuración de las acciones estudiadas no fue un criterio para su elección pues, a causa de lo novedoso del fenómeno y de la debilidad institucional de los municipios, resultaba inútil tratar de evaluar las experiencias en términos de impacto. Su valor reside en ejemplificar lo que está sucediendo: gobiernos locales que irrumpen en el campo de la seguridad ciudadana, la prevención de la violencia y la promoción de la convivencia pacífica como consecuencia de las demandas ciudadanas y no tanto como resultado de la planificación y el convencimiento de las autoridades de que pueden y deben accionar en este campo de forma efectiva, todo ello en un contexto de amplias carencias y predominio de discursos y prácticas represivas.

Tabla 1. Países y municipios incluidos en el proyecto sobre gestión municipal de la seguridad ciudadana en Centroamérica y República Dominicana. Junio de 2009.*

\begin{tabular}{lll}
\hline País & Municipios & \\
\hline Costa Rica & Garabito & Cartago Centro \\
Nicaragua & León & Jinotepe \\
Guatemala & Santa Catarina Pinula & San Juan Sacatepéquez \\
El Salvador & Antiguo Cuscatlán & Santa Tecla** \\
Panamá & San Miguelito & Colón \\
República Dominicana & Distrito Nacional & Villa Bisonó
\end{tabular}

* Aunque Honduras formó parte del estudio, no se pudo evaluar ninguna experiencia concreta debido al golpe de estado perpetrado en ese país el 28 de junio de 2009.

**Se analizó un tercer caso: Ahuachapán.

Fuente: Elaboración propia.

\section{FOCO DE ATENCIÓN}

El estudio partió del reconocimiento de que el Gobierno central es el principal responsable de garantizar la seguridad ciudadana, que el carácter estructural de algunos de los factores asociados al problema (por su naturaleza y magnitud) trasciende las capacidades del gobierno local, por lo que deben ser abordados con políticas nacionales, y que existe una multiplicidad de actores que intervienen en el tema. ${ }^{8}$ Sin embargo, se acentuó el análisis del papel que el gobierno local puede desempeñar en el mejoramiento de la seguridad ciudadana, pues se considera que éste es clave debido a cuatro razones fundamentales. 
Primero, porque es el actor preventivo por excelencia en el territorio, pues es el único que, al aplicar medidas orientadas a mejorar la calidad de vida - recuperar espacios públicos, promover actividades culturales y ordenar el territorio, por ejemplo-, previene la emergencia no sólo de ciertas formas de delito, sino también, y sobre todo, de violencia (Dammert 2007). Segundo, porque al ser el representante del Estado en el territorio es el responsable de impulsar, articular y liderar a los actores y fuerzas sociales presentes en el municipio para establecer un consenso y una estrategia política sobre lo que debe hacerse en el campo de la seguridad y la convivencia (UN-HÁBITAT s/f). Tercero, porque al intervenir a escala local puede identificar las dinámicas específicas de la criminalidad y la violencia, pues de nada sirve conocer la etiología general de estos fenómenos si se desconoce su génesis y expresión en el territorio (Vanderschueren 2000). Y, finalmente, porque en los últimos años, a pesar de las dificultades que han enfrentado los procesos de descentralización y democratización de los gobiernos locales en Centroamérica, se identifica una tendencia regional a que éstos se doten de un mayor número de mecanismos de democracia directa que faciliten el traslado de decisiones políticas municipales a la ciudadanía (PNUD 2008), y además porque se reporta un número creciente de experiencias en el tema de prevención del delito y de la violencia.

\section{LO QUE PUEDE Y DEBE HACERSE}

El conjunto de propuestas que se despliega a continuación no busca asignar nuevas competencias ni funciones a los gobiernos municipales para que asuman tareas que correspondería ejecutar a otras entidades, como por ejemplo la policía. Por el contrario, el esfuerzo radica en identificar aquellas áreas que le son consustanciales y que, si se impulsan y articulan estratégicamente, pueden fortalecer las capacidades municipales para incidir sobre algunos de los factores que anteceden o favorecen el surgimiento de una gran cantidad de hechos violentos. 
REMOZAR Y MODERNIZAR LA ESTRUCTURA MUNICIPAL

Más allá del debate sobre centralización-descentralización y del tema de seguridad ciudadana, una de las tareas más apremiantes que enfrentan los gobiernos locales hoy en día para diseñar y ejecutar políticas públicas idóneas para enfrentar los desafíos del entorno, radica en modernizar y fortalecer sus estructuras. Ello pasa por incrementar las finanzas del gobierno local, sensibilizar y profesionalizar el recurso humano, y fortalecer los canales de participación ciudadana, rendición de cuentas, comunicación y coordinación interna y externa, además de potenciar las capacidades legales del municipio.

El esfuerzo debe estar precedido, eso sí, por voluntad política para impulsar los cambios desde dentro y no sólo para esperar las políticas y acciones del Gobierno central. Es necesario, por tanto, la asignación de partidas presupuestarias y la creación de unidades especializadas en el diseño, ejecución, monitoreo y evaluación de planes, programas y proyectos. Esto es lo que muestran las experiencias que han logrado consolidarse y desarrollar verdaderas políticas de prevención y convivencia, como en el caso del municipio de Santa Tecla en San Salvador.

\section{SUPERAR LA IMPROVISACIÓN}

Uno de los errores más comunes en las iniciativas analizadas es que éstas no responden al diseño de acciones planificadas en el marco de una visión estratégica, sino más bien a demandas y coyunturas específicas, lo que atenta contra su sostenibilidad debido a la fragmentación y aislamiento que las caracteriza.

Por ejemplo, en San Juan Sacatepéquez, Guatemala, el gobierno local tuvo que incursionar en el campo de la seguridad ciudadana obligado por el escenario de conflicto y violencia que produjo la instalación de una empresa productora de cemento en el municipio. Este hecho suscitó la confrontación entre diversos grupos de interés provocando una serie de actos criminales como la imposición de toques de queda por parte de pistoleros privados, asesinatos, secuestros, golpizas y hasta toma de rehenes. Y 
si bien el gobierno local ha jugado un rol importante en la mediación del conflicto a través del diseño de un plan de convivencia y gobernabilidad, es difícil prever que el mismo pueda mantenerse en el tiempo a causa de la debilidad institucional, la fortaleza de los actores en disputa y la ausencia de una estrategia de desarrollo social y participación política que involucre y oriente a los actores en disputa.

En el Distrito Nacional, República Dominicana, la situación es otra: el gobierno local inició acciones en el ámbito de la seguridad ciudadana, específicamente el desarrollo del Observatorio de Violencia al ofrecerle los recursos un organismo internacional. Sin embargo, si no se logra institucionalizar el proyecto, es decir, comprometer recursos propios (personal y presupuesto), lo previsible es que, una vez que se acabe el financiamiento externo, termine también la iniciativa.

En este sentido es menester generar iniciativas que respondan a una visión estratégica que articule las acciones del municipio (coordinación intrainstitucional), involucre a los diversos actores en el territorio, y se oriente a lograr el desarrollo local y promover la convivencia pacífica. Desde esta perspectiva, el tema de la seguridad ciudadana debe ser un componente del plan o estrategia pero no puede constituir el fin último del mismo.

En términos operativos, la estrategia, una vez definida, debe considerar varios aspectos. Por ejemplo, lograr acuerdos mínimos entre los actores que estarán involucrados de una u otra forma en la iniciativa, garantizar la más amplia representación de actores en el proceso, identificar las actividades prioritarias, definir las responsabilidades de quienes participan en su realización, calendarizar las actividades, especificar los recursos existentes o que eventualmente se pueden movilizar, y establecer los métodos de evaluación que se utilizarán.

\section{ESTIMULAR LA PARTICIPACIÓN CIUDADANA}

En relación al punto anterior, es necesario insistir en la utilización de enfoques y metodologías que garanticen una participación efectiva de la ciudadanía en la 
identificación de sus problemas y en la búsqueda de posibles soluciones, ya que sólo de esta manera se logra la inclusión política y el ejercicio activo de la ciudadanía. En este sentido, es impostergable fortalecer las instancias de participación como comités, comisiones y consejos municipales pues, si bien existen formalmente en la mayoría de los municipios analizados, en la práctica no funcionan.

Es preciso, por tanto, diseñar e implementar canales de comunicación efectivos entre el municipio y la ciudadanía: hay que impulsar y encauzar la participación de las y los habitantes a través de campañas de sensibilización e información (estrategia de comunicación de la política) para lograr que se involucren en las diversas fases de las iniciativas impulsadas por el gobierno local (diseño, implementación y evaluación). Pero tales iniciativas constituyen sólo una parte del proceso. Otra parte conlleva la reeducación de los funcionarios para que asuman el proyecto municipal y respondan a las nuevas demandas ciudadanas con vocación de servicio.

Se requiere, por tanto, superar la visión centrada en lo policial, de corte represivo, que tiende a reducir la participación de las y los ciudadanos a informantes o colaboradores de los cuerpos policiales a través de patrullajes vecinales, líneas de denuncia, etc. Se debe avanzar hacia una participación efectiva que garantice la identificación e implicación de la mayoría de los actores presentes en el territorio. De lo contrario, los grupos de interés más fuertes terminarán por imponer sus necesidades —que difícilmente coinciden con las de la comunidad en su conjunto- y ello puede degenerar en la institucionalización de prácticas clientelistas entre tales grupos de interés y el municipio, lo cual alimenta la exclusión política que, de por sí, caracteriza la institucionalidad y la cultura política de la región.

\section{RECUPERAR EL ESPACIO PÚBLICO Y FORTALECER EL CAPITAL SOCIAL ${ }^{9}$}

El cerco de lotes baldíos, la construcción e iluminación de paradas de autobuses, plazas y parques, la recuperación, ampliación y mantenimiento de espacios públicos para el ocio (bibliotecas, plazas de deportes, etc.), así como la regulación urbanística en 
general, son elementos importantes en una estrategia de prevención situacional del delito, sobre todo para mejorar la calidad de vida de las y los habitantes y estimular la apropiación democrática del espacio público. Lo anterior, sin embargo, es insuficiente. Se requieren programas continuos y sostenibles que animen socioculturalmente el espacio público, que desestimulen el aislamiento y la fragmentación social, y que promuevan la convivencia y la solidaridad. De otra forma se corre el riesgo de promover acciones dirigidas al control situacional (vigilancia presencial o electrónica) y de recuperar espacios en las zonas más favorecidas del municipio, lo que reforzaría la exclusión y la estigmatización de ciertos grupos sociales y áreas geográficas.

En este sentido, el impulso y la activación de las redes de convivencia (asociaciones de desarrollo cantonal, comités de prevención de violencia, etc.), la reeducación ciudadana que garantice el cumplimiento de las normas cívicas, así como la promoción de actividades culturales, deportivas y sociales específicamente orientadas al reconocimiento mutuo, la interacción y el pluralismo, son elementos determinantes para que las personas confíen, se asocien y vuelvan a ocupar los espacios públicos.

\section{ACTUAR SOBRE LOS FACTORES DE RIESGO}

Es necesario que el gobierno municipal desarrolle estructuras y mecanismos (observatorios de violencia, por ejemplo) que le permitan identificar y actuar de manera temprana y oportuna sobre los factores de riesgo que vulneran sistemáticamente a determinadas poblaciones y las orillan a una situación conflictiva o violenta, ya sea en el hogar, los centros educativos y laborales, o los espacios públicos. ${ }^{10}$ Para ello se requiere contar con instrumentos sensibles que consideren variables de género, edad, grupo socioeconómico y área geográfica.

Asimismo, el municipio debe trabajar activamente en la regulación y el control efectivo de algunos elementos que propician el surgimiento de conductas violentas, por ejemplo, el expendio de licores, los centros de diversión y los espectáculos públicos. 
En este sentido, y en relación con el punto anterior, resulta útil la promoción de la cultura de paz y la implementación de estrategias no judiciales (como la mediación, la conciliación y el arbitraje) para resolver conflictos menores, como, por ejemplo, disputas vecinales por contaminación sónica, mascotas en descuido, violación del espacio privado, etc. que, de no atenderse oportunamente, podrían desembocar en hechos violentos. Las experiencias analizadas muestran que, aunque se está lejos de transitar por este camino, se han comenzado a ejecutar acciones en esta línea, especialmente en cuanto a la recuperación y ampliación de espacios públicos, y en menor medida a su animación sociocultural. En cuanto al tema de la regulación de patentes, sobre todo en lo que a expendio de licores y espectáculos públicos se refiere, representa todo un desafío para los municipios debido a los intereses económicos en disputa.

\section{INTERVENIR SOBRE LA PERCEPCIÓN}

Reducir el delito y la violencia constituye un objetivo en sí mismo, pero es igualmente relevante trabajar sobre las percepciones, puesto que muchas veces éstas, además de estar bastante alejadas de la realidad, motivan prácticas contrarias a la tolerancia y al respeto hacia las instituciones democráticas. Por ello, es vital que el gobierno local disponga de información válida y confiable dirigida a conocer, de manera rigurosa, el comportamiento violento y criminal del municipio con el fin de desplegar campañas de información y educación que desestimulen los estereotipos y el rechazo a grupos y zonas que han sido particularmente estigmatizados, lo cual refuerza los procesos de fragmentación y exclusión social, así como el desencadenamiento de acciones que alimentan el círculo de la violencia.

\section{MEJORAR LA COORDINACIÓN}

Ya se ha mencionado que el gobierno municipal no es el único responsable de mejorar la seguridad ciudadana en el municipio: es uno entre muchos actores. Sin embargo, éste debe asumir un papel protagónico dado que es el representante del Gobierno en el territorio. Por tanto, una de sus tareas fundamentales, además de implementar y diseñar 
iniciativas, es la de promover y liderar coaliciones que articulen la mayor cantidad de actores y fuerzas vivas del territorio.

Es primordial que el municipio cuente con un mapa de actores locales vinculados al desarrollo social, la prevención de la violencia y la promoción de la paz social, ya que este instrumento facilita el conocimiento y la identificación de los recursos disponibles en el territorio, optimiza su uso y evita duplicaciones. De igual forma, es necesario conocer los espacios de coordinación interinstitucional para aprovechar las fortalezas y superar las deficiencias o vacíos existentes (PNUD 2010).

Lamentablemente, ambos puntos están muy lejos de operar en la realidad y más bien lo que se identifica como constante es la ausencia de fuentes de información válidas, confiables y actualizadas en las municipalidades, así como la poca o escaza articulación de esfuerzos entre éstas y los demás actores presentes en el territorio, lo cual termina por debilitar el impacto y la sostenibilidad de las acciones desarrolladas.

En términos de coordinación intersectorial hay dos aspectos centrales por destacar. En primer lugar, la municipalidad debe conocer las acciones que realizan las instituciones nacionales en el territorio, por ejemplo la policía, los tribunales y los ministerios de justicia, para identificar formas de cooperación y coordinación. Un caso interesante en esta línea son los programas de sanciones alternativas que implican trabajo comunitario. En segundo lugar, es necesario vigilar y garantizar la coherencia de las acciones del municipio con las políticas nacionales. El problema, sin embargo, es que la mayoría de los países carecen de una verdadera política de desarrollo y convivencia. Esta laguna no implica que la municipalidad no tenga la obligación de derivar a nivel nacional las responsabilidades de otras políticas, como, por ejemplo, transporte, empleo y educación. 


\section{LO QUE NO DEBE HACERSE}

\section{LIMITAR EL PAPEL DEL GOBIERNO MUNICIPAL AL ÁMBITO DE LA REPRESIÓN}

El análisis de las experiencias concretas permitió identificar que en la mayoría de los gobiernos locales estudiados domina una perspectiva de la función municipal restringida a los temas de seguridad ciudadana y convivencia pacífica. El énfasis se deposita en el control situacional de ciertos delitos y esto es justamente lo que explica el interés generalizado por crear o fortalecer las policías municipales. De hecho, en casi todos los municipios estudiados se registra la presencia de cuerpos de esta índole, con el agravante de que, en algunos casos, se les asignan tareas represivas — como participar en arrestos - más que preventivas — patrullajes_ o de coordinación con los cuerpos de policía nacional.

Esta situación se torna problemática por varias razones. Primero, porque se asigna a dichos cuerpos funciones y expectativas que no pueden cumplir. Afirmar que pueden resolver por sí solos el problema de la inseguridad ciudadana o sustituir a la policía nacional es otorgarles tareas que no les corresponden. En segundo lugar porque, además de entrañar el riesgo de menoscabar lo que puede realizar el gobierno local en cuanto a prevención del delito y otras formas de violencia, limita la participación ciudadana al generar entre los habitantes el temor de quedar reducidos, bajo este esquema, a informantes de la policía. Y esto es sumamente nefasto en el contexto político e histórico de las sociedades centroamericanas por el papel que dichos cuerpos desempeñaron durante los conflictos armados. Por último, se trata de un servicio muy oneroso que sólo podrían costear las municipalidades de mayores ingresos, lo cual acentúa la brecha en la prestación de este servicio público llamado seguridad ciudadana. Además, también genera un contrasentido, ya que ciertos gobiernos locales (representantes de lo público por excelencia) comienzan a vender seguridad y, por ende, a fragmentar y privatizar el servicio. 
En este sentido, es necesario iniciar el debate sobre la función que deben cumplir las policías municipales. Desde la perspectiva de este trabajo, éstas deberían circunscribirse a proteger los bienes municipales, vigilar la ejecución de las ordenanzas del gobierno local, coordinar con los cuerpos de policía nacional y depender de éstos en términos funcionales, es decir, someterse a su mandato. Esto es justamente lo que sucede en el caso de Nicaragua, donde las policías municipales dependen administrativamente de las municipalidades pero funcionalmente de la Policía Nacional.

\section{DESAFÍOS Y PERSPECTIVAS}

\section{FORTALECER LA PREVENCIÓN}

En el contexto actual, los discursos y las prácticas dominantes alrededor del tema de la seguridad ciudadana son marcadamente estrechos, autoritarios e ineficaces. Se homologa seguridad ciudadana con represión de delitos específicos. Los gobiernos municipales no escapan a esta tendencia y, si bien en el plano discursivo hay un reconocimiento de que se está ante un problema social complejo, en la práctica las acciones realizadas — cuando las hay — dan cuenta de una perspectiva reduccionista e intolerante.

Por lo señalado, se torna necesario sensibilizar a las autoridades y funcionarios municipales para que tomen conciencia, tanto de la magnitud del problema y de la responsabilidad de contribuir junto con otros actores al mejoramiento de la seguridad ciudadana, como de garantizar el desarrollo social y humano de las personas que habitan en el territorio. Esta premisa advierte sobre lo inadecuado de apostar casi de forma exclusiva por aspectos represivos como la creación de policías municipales, pues, si no se actúa sobre los factores estructurales asociados al incremento de ciertas formas de violencia, es poco lo que puede lograrse para reducir su incidencia.

Otra área en la que se impone el trabajo de las municipalidades es en el diseño de programas orientados al servicio comunitario. Ello brindaría un soporte considerable 
para la ejecución de medidas alternativas a la prisión y daría un impulso significativo al cumplimiento de medidas de rehabilitación y reinserción social, así como a la implementación de programas de educación ciudadana y de opciones laborales para grupos particularmente vulnerables como los jóvenes.

Se requiere, entonces, una política nacional y local que articule esfuerzos para brindar a las personas oportunidades efectivas para su desarrollo pleno. Éste es el verdadero origen de una convivencia pacífica, que requiere como uno de sus soportes —no el único, claro está- al gobierno local. Y esto parece factible en el contexto actual, en el que los discursos sobre seguridad que se centran en las personas y privilegian los aspectos comunitarios de la prevención han ganado terreno a pesar del predominio de la solución penal.

\section{AVANZAR EN LA CONSTRUCCIÓN DE UN ENFOQUE INTEGRADOR}

Es necesario trabajar en la construcción conceptual y metodológica de un esquema de políticas públicas que dé sustento al trabajo que actualmente realizan las municipalidades en los campos de la seguridad ciudadana, la prevención de la violencia y la promoción de la convivencia pacífica. Esto implica una discusión teórica y una aplicación práctica. Por ello, debe ser un trabajo conjunto entre academia y autoridades municipales. Dicho esquema exige al menos dos requisitos: respetar los derechos humanos y la institucionalidad democrática, y ser sensible al contexto y a las circunstancias de cada municipio para evitar que se convierta en un recetario con medidas estándar destinadas al fracaso.

\section{FORTALECIMIENTO MUNICIPAL}

Este punto resulta sumamente difícil de lograr en sistemas políticos como los centroamericanos, donde aún prevalecen el centralismo y una débil institucionalidad en lo local y lo nacional. La tarea resulta aún más compleja si se toma en cuenta la necesidad de estimular las instancias de participación ciudadana, donde la cultura democrática es tan débil e inestable como la institucionalidad que la soporta, lo cual 
favorece la corrupción y el oportunismo político. A pesar de ello, no hay que perder de vista que ya hay municipios donde los temas de la seguridad y la convivencia se han desarrollado plenamente y con resultados satisfactorios. Éste es el caso de varios municipios en El Salvador y Nicaragua, por señalar sólo dos ejemplos.

\section{CREACIÓN DE UN DIRECTORIO DE EXPERIENCIAS}

Uno de los mayores vacíos en este tema es la ausencia de un directorio de experiencias para poder conocer cuántos y cuáles municipios toman iniciativas en este campo y en qué consisten tales iniciativas. Es decir, se requiere producir un inventario de experiencias y proyectos para contar con información actualizada y confiable sobre lo que están haciendo los municipios de la región en el campo de la convivencia. Por ejemplo, en Costa Rica, la municipalidad de Desamparados cuenta con una Casa de los Derechos de los Migrantes, un tema central para la promoción de los valores de tolerancia y convivencia pacífica en el marco de la institucionalidad democrática. Pero esa experiencia, al igual que muchas otras en la región, no se encuentra debidamente registrada ni sistematizada, lo que dificulta que reciban mayor apoyo y que la iniciativa pueda trasladarse a otros contextos.

\section{INTEGRAR UNA RED CENTROAMERICANA Y DEL CARIBE DE GOBIERNOS MUNICIPALES}

Es claro que la violencia que se manifiesta en Centroamérica varía, no sólo en función de aspectos como sexo, edad y género, sino también, y sobre todo, por la variable territorio. Esta última ocasiona que la problemática de un municipio en El Salvador, por ejemplo, se parezca más a la de un municipio de Panamá o Costa Rica, que a la de otros territorios de El Salvador. Por tanto, es necesario constituir una liga regional de municipios que promueva el intercambio constante de experiencias e iniciativas, que acerque a quienes enfrentan problemáticas similares y que proporcione las herramientas necesarias para trasladar experiencias. 


\section{ANEXO 1}

Cuadro A1. Centroamérica. Denuncias por homicidio por cada 100,000 habitantes (2000 a 2008)

\begin{tabular}{|l|c|c|c|c|c|c|c|c|c|}
\hline \multirow{2}{*}{ Países } & \multicolumn{7}{|c|}{ Homicidio } \\
\cline { 2 - 11 } & \multicolumn{9}{|c|}{ Años } \\
\cline { 2 - 11 } & $\mathbf{2 0 0 0}$ & $\mathbf{2 0 0 1}$ & $\mathbf{2 0 0 2}$ & $\mathbf{2 0 0 3}$ & $\mathbf{2 0 0 4}$ & $\mathbf{2 0 0 5}$ & $\mathbf{2 0 0 6}$ & $\mathbf{2 0 0 7}$ & $\mathbf{2 0 0 8}$ \\
\hline El Salvador & 37,3 & 34,5 & 31,1 & 32,7 & 41,0 & 55,0 & 56,2 & 49,2 & 44,0 \\
\hline Guatemala & 28 & 30 & 32 & 37 & 38 & 44 & 47 & 45 & 48 \\
\hline Nicaragua & 9,6 & 10,6 & 10,7 & 12,1 & 12,0 & 13,4 & 13,1 & 12,8 & 13,0 \\
\hline Costa Rica & 6,4 & 6,4 & 6,3 & 7,3 & 6,7 & 8,0 & 8,2 & 8,0 & 11,0 \\
\hline Panamá & 10,1 & 10,2 & 12,4 & 10,9 & 9,7 & 11,3 & 11,3 & 13,0 & 12 \\
\hline República Dominicana & 13,1 & 12,5 & 14,5 & 18,7 & 25,3 & 26,4 & 23,6 & 21,9 & 25,1 \\
\hline
\end{tabular}

Fuente: Crimen e inseguridad. Indicadores para las Américas. Proyecto Violencia y Criminalidad en América Latina y el Caribe. FLACSO Chile, 2010.

Cuadro A2. Centroamérica. Denuncias por robo por cada 100,000 habitantes (2000 a 2008)

\begin{tabular}{|l|c|c|c|c|c|c|c|c|c|}
\hline \multirow{2}{*}{ Países } & \multicolumn{9}{|c|}{ Robo } \\
\cline { 2 - 10 } & \multicolumn{9}{|c|}{ Años } \\
\cline { 2 - 11 } & $\mathbf{2 0 0 0}$ & $\mathbf{2 0 0 1}$ & $\mathbf{2 0 0 2}$ & $\mathbf{2 0 0 3}$ & $\mathbf{2 0 0 4}$ & $\mathbf{2 0 0 5}$ & $\mathbf{2 0 0 6}$ & $\mathbf{2 0 0 7}$ & $\mathbf{2 0 0 8}$ \\
\hline El Salvador & 168,9 & 120,7 & 81,8 & 69,0 & 77,7 & 84,7 & 83,4 & n.d & n.d \\
\hline Guatemala & 174,4 & 160,6 & 161,3 & 165,7 & 158,6 & 142,5 & 119,3 & n.d & n.d \\
\hline Nicaragua & 214,3 & 228,3 & 235,5 & 232,9 & 226,3 & 218,3 & 249,1 & 288,4 & 318,7 \\
\hline Costa Rica & 337,5 & 287,1 & 268,3 & 279,2 & 311,0 & 341,0 & 357,7 & 338,9 & n.d \\
\hline Panamá & n.d & n.d & n.d & n.d & 155,2 & 183,2 & n.d & n.d & n.d \\
\hline República Dominicana & 411 & 343 & 273 & 309 & 228,2 & 172,8 & 161,6 & 464,1 & 305,0 \\
\hline
\end{tabular}

Fuente: Crimen e inseguridad. Indicadores para las Américas. Proyecto Violencia y Criminalidad en América Latina y el Caribe. FLACSO Chile, 2010. 


\section{BIBLIOGRAFÍA}

Calderón, Rodolfo, 2010, «Delito y cambio social en Costa Rica», en Política criminal en el Estado social de derecho, editado por Llobet, Javier y Douglas Durán, Editorial Jurídica Continental, Costa Rica.

Dammert, Lucía, 2007, «Seguridad pública en América Latina: ¿qué pueden hacer los gobiernos locales?», en Revista Nueva Sociedad, n. 212, noviembre-diciembre, Buenos Aires, pp. 67-81.

ERIC (Equipo de Investigación, Reflexión y Comunicación de Honduras), IDESO, IDIES, IUDOP, 2001, Maras y pandillas en Centroamérica, v. 1, UCA, Managua.

FLACSO Chile, 2010, Crimen e inseguridad. Indicadores para las Américas, Proyecto Violencia y Criminalidad en América Latina y el Caribe, FLACSO Chile, BID, Santiago de Chile.

Inter-American Development Bank (IDB), 2007, Outsiders?: The Changing Patterns of Exclusion in Latin America and the Caribbean, IDB, Washington, D. C.

Kliksberg, Bernardo, 2007, Mitos y realidades sobre la criminalidad en América Latina, F\&G Editores, Guatemala.

__, 2000, Capital social y cultura: claves olvidadas del desarrollo, BID, Argentina.

Londoño, Juan, Luis Alejandro Gaviria y Rodrigo Guerrero, 2000, Asalto al desarrollo: violencia en América Latina, BID, Washington, D. C.

Matul, Daniel, 2007, «Reflexiones iniciales sobre los gobiernos locales, la seguridad ciudadana y las políticas públicas locales: explorando sus vínculos», en Encuentro 
Regional: hacia una política municipal de seguridad ciudadana, ciudadanía y pertenencia, Fundemuca, San José.

Programa de Naciones Unidas para el Desarrollo, 2010, Ciudades seguras: el ABC de la convivencia y la seguridad ciudadana. Herramientas para la gestión local, PNUD, El Salvador.

—_, 2009, Informe sobre desarrollo humano para América Central: abrir espacios a la seguridad ciudadana y el desarrollo humano, PNUD, AECID, SICA, Bogotá.

—_, 2008, Informe estado de la región, PNUD, Costa Rica.

—, 2005, Informe sobre desarrollo humano nacional. El temor: (in) seguridad ciudadana y desarrollo humano en Costa Rica, PNUD, Costa Rica.

UN-HÁBITAT, s/f, Un enfoque democrático en materia de seguridad urbana, en http://www.onuhabitat.org/index.php?option=com_docman\&task=doc_details\&Itemid= 235\&gid=29 [consulta: 12 de enero de 2011].

Vanderschueren, Franz, 2000, «Seguridad ciudadana: solidaridad democrática y prevención», en Conversaciones públicas para ciudades más seguras, coordinado por Hugo Acero, Ediciones SUR, Santiago de Chile, pp. 130-138.

Zinecker, Heidrun, 2010, «Gewalkriminalität in Zentralamerika Entwurfeineserklärenden Theorie-Modells», en Gewalkriminalität in Zentralamerika: Formen, Ursachen, Einhegungsmöglichkeiten, editado por Saffer, K. y Heidrun Zinecker, Nomos, Alemania. 


\section{NOTAS}

${ }^{1}$ Los resultados generales de la investigación sobre la que da cuenta este artículo fueron publicados por FLACSO-Costa Rica bajo el título: Ciudades seguras en Centroamérica y República Dominicana: acciones estratégicas desde el gobierno local. (FLACSO-CR 2010).

${ }^{2}$ Ver Cuadros A1 y A2 del Anexo 1.

${ }^{3}$ El surgimiento de un enfoque como éste sólo puede entenderse, para el caso centroamericano, en el marco de los procesos de pacificación. En términos de seguridad significó remplazar — como eje de atención principal — la defensa del territorio frente a amenazas externas (doctrina de seguridad nacional), por la atención y la protección de las personas (Matul 2007).

${ }^{4}$ Se debe recordar, en este sentido, la presencia de otros actores como las empresas privadas de seguridad, que actúan en sintonía con las visiones punitivas que actualmente dominan en el tratamiento del tema y que por ende no contribuyen a reducir el problema sino a agravarlo.

${ }^{5}$ El telón de fondo para entender la participación de los gobiernos locales de la región en el tema de la seguridad, sobre todo a partir de la segunda mitad de los años noventa, está marcado por los procesos de descentralización estatal, así como por una reacción del Estado central que procura compartir responsabilidades frente al incremento del delito, las demandas ciudadanas y su incapacidad de proponer respuestas efectivas.

${ }^{6}$ El proyecto de investigación se denominó Gestión Municipal de la Seguridad Ciudadana en Centroamérica y República Dominicana, desarrollado y coordinado por FLACSO-Costa Rica con el apoyo técnico de FUNDEMUCA y el auspicio de la Agencia Española de Cooperación Internacional para el Desarrollo (AECID), en el marco de una iniciativa mayor impulsada por FLACSO-Secretaría General para estudiar la gobernabilidad democrática y la convivencia pacífica en América Latina.

${ }^{7}$ Se entiende por "«violencia» el uso o amenaza de uso de la fuerza física o psicológica con el fin de causar daño o doblegar la voluntad. El «despojo» se refiere al acto de privar ilegítimamente de su patrimonio a una persona física o jurídica (PNUD 2005:35).

${ }^{8}$ Dos ejemplos claros de problemas estructurales que desbordan las capacidades municipales y que deben ser atendidos a través de políticas nacionales son la debilidad del sistema de justicia penal y la exclusión socioeconómica que afecta a un sector importante de la sociedad, pues se reconoce que ambos elementos están en la base de un conjunto importante de hechos violentos, ya sea para satisfacer intereses materiales o necesidades de protección y justicia (IDB 2007).

${ }^{9}$ En términos generales, el capital social puede ser entendido, para los propósitos de este trabajo, como el grado de confianza entre los actores sociales de una sociedad, las normas de comportamiento cívicas practicadas y el nivel de asociatividad que la caracterizan (Kliksberg 2000).

${ }^{10}$ El conflicto es consustancial a la interacción humana, por tanto, se impone el diseño de mecanismos institucionales que posibiliten su abordaje y resolución por vías pacíficas.

Fecha de recepción: 23 de marzo de 2011.

Fecha de aceptación: 8 de julio de 2011. 